\title{
Effect of H7N1 vaccination on highly pathogenic avian influenza H7N7 virus transmission in turkeys
}

\author{
Marian E.H. Bos ${ }^{\mathrm{a}, *}$, Mirjam Nielen ${ }^{\mathrm{a}}$, Guus Koch $^{\mathrm{b}}$, Arjan Stegeman ${ }^{\mathrm{a}}$, Mart C.M. De Jong ${ }^{\mathrm{c}}$ \\ a Department of Farm Animal Health, Faculty of Veterinary Medicine, Utrecht University, Yalelaan 7, 3584 CL Utrecht, The Netherlands \\ ${ }^{\mathrm{b}}$ Avian Virology, Department of Virology, Central Veterinary Institute of Wageningen UR, P.O. Box 65, 8200 AB Lelystad, The Netherlands \\ ${ }^{\mathrm{c}}$ Department of Quantitative Veterinary Epidemiology, Animal Sciences Group, P.O. Box 338, 6700 AH Wageningen, The Netherlands
}

\section{A R T I C L E I N F O}

\section{Article history:}

Received 29 February 2008

Received in revised form 4 September 2008

Accepted 4 September 2008

Available online 24 September 2008

\section{Keywords:}

Highly pathogenic avian influenza

Vaccination

Transmission experiment

Turkeys

\begin{abstract}
A B S T R A C T
This study describes the results of a transmission experiment with highly pathogenic avian influenza (HPAI) H7N7 virus in 12-week-old turkeys. Cloacal and tracheal swabs as well as serum samples were taken to monitor the infection both in inoculated and in susceptible contact turkeys, which were all either unvaccinated, vaccinated once or vaccinated twice with H7N1. Swabs were tested by real-time RT-PCR and serum samples with hemagglutination inhibition test $(\mathrm{HI})$. Unvaccinated contact birds had a mean infectious period of 6.2 days, and an estimated transmission rate parameter of 1.26 per infectious bird per day. However, no virus shedding was found in inoculated vaccinated turkeys and thus we concluded that vaccination with H7N1 protected against challenge with HPAI H7N7 virus.
\end{abstract}

(C) 2008 Elsevier Ltd. All rights reserved.

\section{Introduction}

During the last decades, highly pathogenic avian influenza (HPAI) viruses have caused outbreaks of AI in poultry worldwide [1]; in certain regions HPAI has even become endemic (e.g. Southeast Asia). Vaccination is considered an important tool in the prevention and control of AI. A field trial with H5N2 vaccine in Hong Kong in 2002 showed that vaccinated chickens were protected from disease, and HPAI H5N1 virus transmission was reduced [2]. The same was concluded for vaccination with H5N1 and H5N2 vaccines in Vietnam (2005) [3] and Mexico (1995) [4], although due to the various control strategies and vaccines implemented during these epidemics it was not possible to claim a direct relationship between reduced transmission and the applied vaccination strategy. The latter is a common problem in field studies, because many factors, e.g. the applied vaccination scheme [5], may influence the result.

Transmission experiments are suitable for studying the effect of vaccination on virus transmission in general [6]. Such experiments are performed under controlled settings, enabling to quantify the effect of different parameters on transmission; for example the host species, the infectious dose, the virus strain, and, especially,

\footnotetext{
* Corresponding author. Tel.: +31 30253 6487; fax: +31 302521887 E-mail address: m.e.h.bos@uu.nl (M.E.H. Bos).
}

vaccination. Transmission experiments studying the effect of vaccination on transmission of HPAI virus have been carried out in chickens [7], pheasants and ducks [8] with H7N7 HPAI virus. These studies showed that indeed the same virus can have different transmission parameters in different host species, and also showed that the effect of vaccination on transmission can vary from one species to another

Turkey meat production is important in Europe, North- and Central America [9] and therefore large turkey stocks are present on those continents. Recent HPAI outbreaks in turkey flocks occurred in Italy (1999; H7N1 [10]) and Chile (2002; H7N3 [11]), and various LPAI outbreaks have been detected in, e.g. US, Italy and Great Britain [12]. Consequently, turkeys may play an important role in AI outbreaks and an effective control strategy in turkeys is essential in attempts to eliminate HPAI. From the literature it appears that turkey flocks have more new cases of HPAI H7N1 per 1000 flocks per day at risk compared to other bird species, such as game birds, water fowl and layer hens [10]. Moreover, a challenge study showed that a minimal nasal infection dose of HPAI H5N9 was fatal to turkeys, whereas chickens sometimes recovered or were not affected at all [13]. Although vaccination of turkeys has been applied in the control of LPAI in Italy [5,14] it is unknown whether vaccination can stop transmission of HPAI virus among turkeys.

Therefore, the main goal of this study was to quantify the effect of vaccination with H7N1 on HPAI H7N7 virus transmission in turkeys. 


\section{Materials and methods}

\subsection{Turkeys}

Sixty four-week-old female turkeys were obtained from a commercial farm, since no SPF turkeys were available. During the experiment the turkeys were housed and handled in a high containment unit at the Central Veterinary Institute of Wageningen UR under BSL3+ conditions. They were loose housed on floors with wood shavings, fed ad libitum, and had free access to drinking water. The experiments were reviewed by an animal ethics committee and the experiments complied with the Dutch law on animal experiments. The birds were negative in the hemagglutination inhibition test $(\mathrm{HI})$ for antibodies against $\mathrm{H} 7$ and negative in the immunofluorescence test (IF) for antibodies against N7 before the challenge. Clinical signs of the birds were recorded daily and the birds were euthanized for animal welfare reasons if it was expected that they would not survive until the next sampling time.

\subsection{Challenge virus}

Turkeys were challenged by inoculation with highly pathogenic avian influenza H7N7 virus, obtained on the index farm of the Dutch epidemic in 2003 (A/Chicken/Netherlands/621557/03). The intravenous pathogenicity index (IVPI) of the virus was determined to be 2.9 as described in ref. [15]. The turkeys were challenged both intranasally $(0.1 \mathrm{ml})$ and intratracheally $(0.1 \mathrm{ml})$ with diluted allantoic fluid containing $10^{6}$ median egg infectious dose $\left(\operatorname{EID}_{50}\right) / \mathrm{ml}$.

\subsection{Vaccine}

Turkeys were vaccinated with an inactivated oil-emulsion vaccine, which was commercially available and contained H7N1 antigen (A/Chicken/Italy/99) [7]. The dosage was $0.5 \mathrm{ml}$ subcutaneously, as recommended by the manufacturer. The hemagglutinin-antigen content was $45 \mu \mathrm{g} / \mathrm{ml}$ vaccine, which was determined as described in Van der Goot et al. [7]. In short: viral proteins were extracted from the oil-emulsion vaccine, and analysed by electrophoresis. The gel was stained with SYPRO orange dye (Molecular Dynamics) and scanned. Staining of the hemagglutinin band was compared with the staining of BSA at different loading concentrations as standard. The homology between the HA1, the immunogenic part, of the vaccine and challenge virus was $98 \%$ [7].

\subsection{Experimental setup}

The birds were randomly divided into three parallel groups of 20 turkeys based on their wing clip identification number (group 0 : 1-20, group 1: 21-40, group 2: 41-60). The control group (group 0 ) did not receive vaccination. Group 1 was vaccinated once at 10 weeks of age and the third group (group 2) was vaccinated twice; at 6 weeks and at 10 weeks of age. The three treatment groups were then each divided into two groups of 10 animals (subgroups $A$ and B), to form two replicates per treatment. Each subgroup was housed in a separate room (10 turkeys $/ 9 \mathrm{~m}^{2}$ ). From each subgroup five contact animals (C) were housed separately before the challenge. The other five birds (I) were inoculated at 12 weeks of age. The contact birds were placed back with the inoculated birds in the same room the day after the challenge (therefore I:C was 5:5 [6]). At day 28 post challenge (p.c.) all remaining live birds were euthanized and the experiment was terminated.

\subsection{Serology}

Blood samples were collected from all birds at 6 weeks of age, and subsequently weekly from all vaccinated turkeys. Post challenge all turkeys were sampled weekly. Sera were separated and stored at $-20^{\circ} \mathrm{C}$ until analysed. Sera were incubated for $30 \mathrm{~min}$ at $56^{\circ} \mathrm{C}$ to inactivate the sera for further analysis outside of BSL3+ restrictions.

HI was used to detect antibodies against $\mathrm{H7}$ in the serum. In short: the sera were absorbed with $20 \%$ chicken erythrocytes ( $1: 1$ dilution) and left overnight at $4{ }^{\circ} \mathrm{C}$. The sera were tested in V-bottom 96-wells micro titre plates using 8 units of HA antigen and $1 \%$ chicken erythrocytes [15]. Results are given as ${ }^{2} \log$ titre.

The sera of day 0 and 28 p.c. were tested with an IF for presence of antibodies against N7, to test whether the birds were fully challenged. The protocol followed the method as proposed by Cattoli et al. [16], with some modifications. In short: monolayers of SF21 insect cells were grown in 96-microwell plates and when confluent infected with N7-recombinant baculovirus. Fixed monolayers were incubated with sera diluted to 1:50 and 1:250 dilutions in a high salt buffer containing $4 \%$ horse serum. Next the plates were washed and subsequently incubated for $1 \mathrm{~h}$ at $37^{\circ} \mathrm{C}$ in a dry incubator. The plates were washed again and chicken conjugate $(\mathrm{RaCh} / \mathrm{IgG}(\mathrm{H}+\mathrm{L}) / \mathrm{FITC}$, Nordic) was added followed by another incubation period of $1 \mathrm{~h}$ at $37^{\circ} \mathrm{C}$. Finally, the plates were washed and filled with PBS, after which the results could be read.

\subsection{Virus detection}

From the challenge onwards cloacal and tracheal swabs were collected daily until 10 days p.c., hereafter swabs were taken every 3/4 days until the experiment ended at 28 days p.c. The swabs were stored at $-70^{\circ} \mathrm{C}$ in $2 \mathrm{ml} 2.95 \%$ tryptose phosphate buffer with penicillin and streptomycin until analysed. Next, swabs were analysed with a quantitative real-time RT-PCR (reverse transcriptase-polymerase chain reaction). RNA was extracted from $200 \mu \mathrm{l}$ swab fluid using $300 \mu \mathrm{l}$ external lysis buffer from a commercially available kit and machine (MagNA Pure LC ${ }^{\circledR}$ Total Nucleic Acid Isolation Kit and MagNA Pure LC ${ }^{\circledR}$ Instrument; Roche $\left.{ }^{\circledR}\right)$. The procedure yielded $50 \mu$ l isolated RNA per swab, which was stored at $-70^{\circ} \mathrm{C}$.

The real-time RT-PCR procedure required $5 \mu \mathrm{l}$ of isolated RNA to which a mix was added according to the instructions of the manufacturer (OneStep RT-PCR kit; Qiagen ${ }^{\circledR}$ ). This mix contained amongst others a Taqman probe (AI-MP-TqMn1) and a forward and reverse primer (AI-MpFW45 and AI-MpRv251, respectively; sequences can be found in Table 1 ) targeting the M-gene of the virus. ROX was used as reference dye. The PCR reaction was performed using the following protocol in a $\mathrm{Mx} 4000^{\mathrm{TM}}$ (Stratagene ${ }^{\odot}$ ): $30 \mathrm{~min}$. at $50^{\circ} \mathrm{C}, 15 \mathrm{~min}$. at $95^{\circ} \mathrm{C}$ and then 45 cycles of $5 \mathrm{~s}$. at $95^{\circ} \mathrm{C}, 15 \mathrm{~s}$. at $58^{\circ} \mathrm{C}$ and $20 \mathrm{~s}$. at $72^{\circ} \mathrm{C}$, after which the hold-stage was entered (at $\left.25^{\circ} \mathrm{C}\right)$.

Results are given in ${ }^{10} \mathrm{log}$ virus titre (expressed as equivalent units of $\mathrm{EID}_{50} / \mathrm{ml}$ ). A standard curve $\left({ }^{10} \mathrm{log}\right.$ virus titre vs. CT (threshold cycle)-value) was prepared for each swab batch by analysing a

\section{Table 1}

Sequences of probe and primers used in the real-time RT-PCR procedure

\begin{tabular}{lll}
\hline Material & Name & Sequence \\
\hline Taqman Probe & AI-MP-TqMn1 & $\begin{array}{l}\text { 5'-6FAM-CTCAAAGCCGAGATCGCGCAGA- } \\
\text { XT-PH-3' }\end{array}$ \\
Forward primer & AI-MpFW45 & 5'-CTTCTAACCGAGGTCGAAACGTA-3' \\
Reverse primer & AI-MpRv251 & 5'-CACTGGGCACGGTGAGC-3' \\
\hline
\end{tabular}


10-fold dilution of H7N7 virus with a known titre. A swab was considered positive when the value read on the standard curve was larger than the lowest detectable ${ }^{10} \log$ virus titre value of the standard curve. Single positive swabs (i.e. swabs of the same bird of the days before and after were negative) were repeated in the realtime RT-PCR, and were considered positive only when the repeat was also positive.

\subsection{Statistical analysis}

A generalized linear modelling method was used to analyse the results of the transmission experiment and estimate the transmission rate parameter $(\beta)$. To that end, the data were transformed into a $S I$-format with $S$ denoting the number of susceptible birds present per day and $I$ the number of infectious birds present per day. The total number of birds present per day was denoted by $N$. Contact birds were considered to have been infected the day before their first positive tracheal and/or cloacal swab and therefore were denoted case $(C)$ on that day.

The GENMOD procedure from the SAS ${ }^{\circledR}$ program (SAS Institute Inc., 2002) was used to program the model. The response variable $C$ was assumed to be binomially distributed, with $S$ as binomial total. Thus the variable $C / S$ was analysed using binomial distribution with a complementary log-log-link and the offset $\log (I / N)$. Hence, cases on one day are caused by the infectious birds present during that day. The replicate groups were inserted as repeated subject. It is assumed that within each treatment all susceptible birds are equally susceptible, all infectious birds are equally infectious, and that each infectious bird is equally infectious over time. Furthermore, it is assumed that each infected bird constitutes an independent risk to infect a susceptible bird. $\beta$ follows from the intercept of the model, and subsequently the reproduction ratio $R_{0}$ can be estimated by taking the product of the estimated $\beta$ and the estimated mean length of the infectious period, with the latter
Inoculated
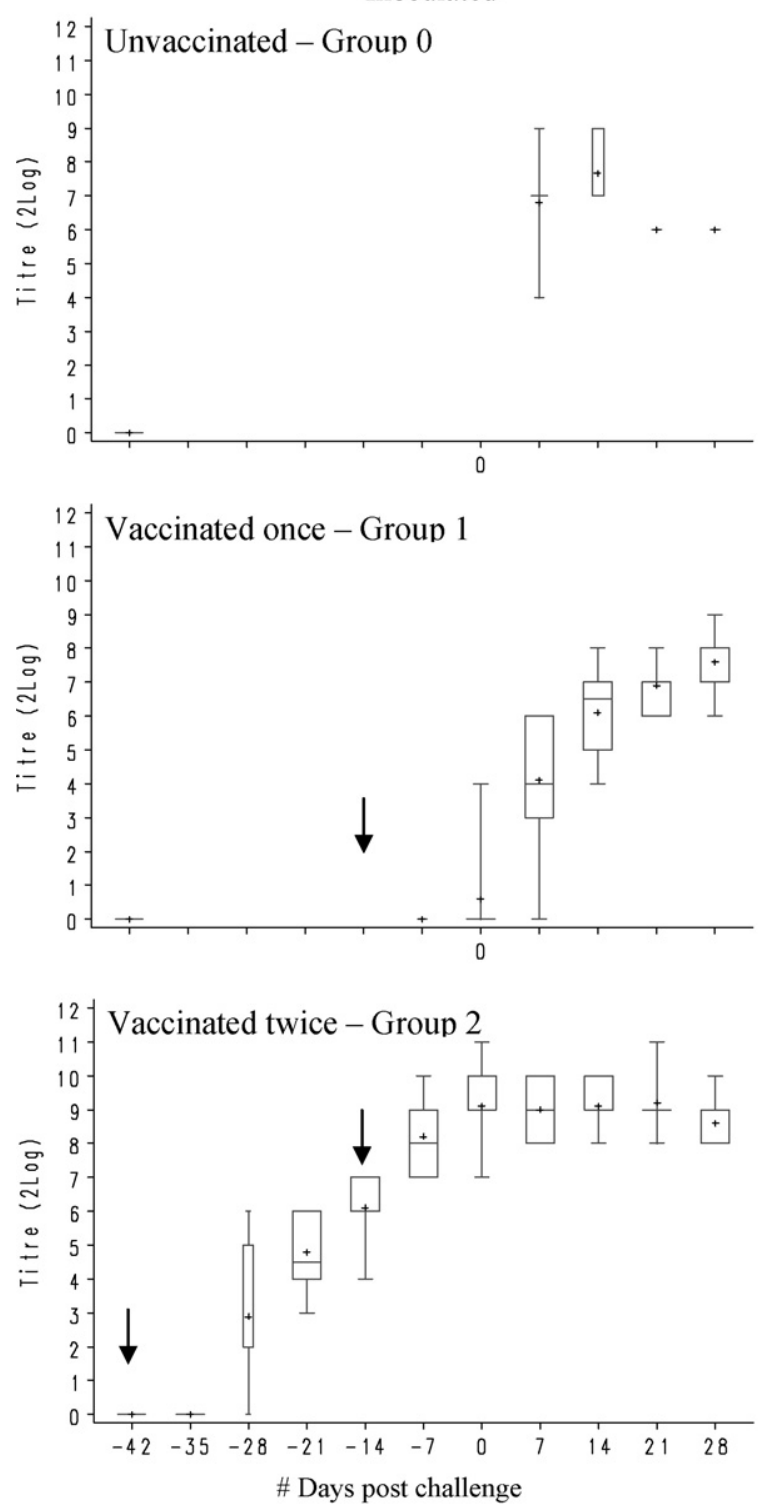

Contact
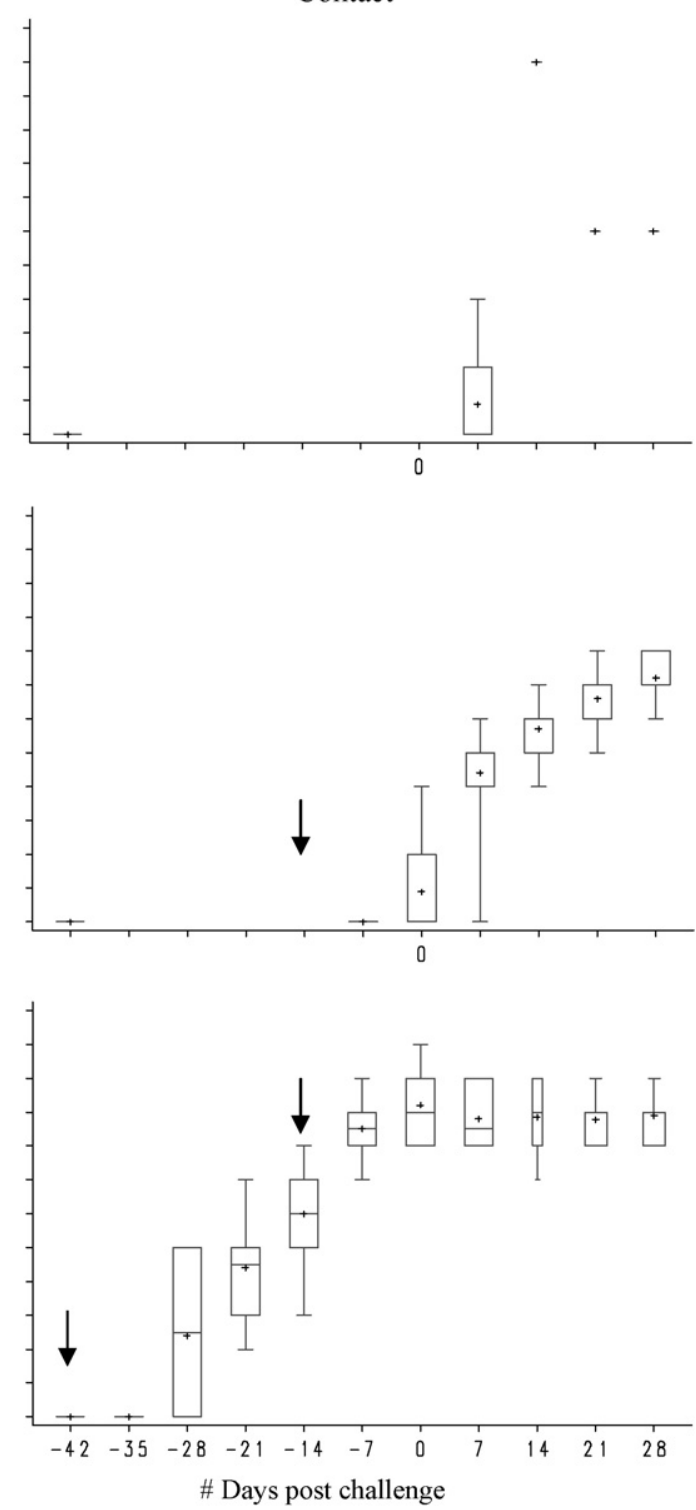

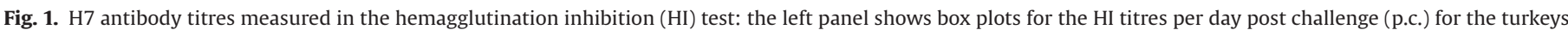

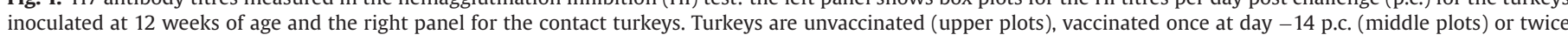

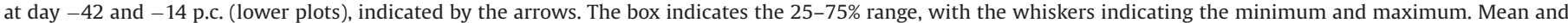
median are given by the plus marker and line inside the box, respectively, and the width of the box indicates the number of observations included (maximum $=10$ ). 
directly observed from the unvaccinated contact turkeys $[17,18]$. Virus shedding was compared between inoculated unvaccinated and vaccinated birds by means of a Fisher's exact test, and the NPAR1WAY procedure in SAS ${ }^{\circledR}$ was used to compare HI antibody titres between groups. Sensitivity analysis was performed on the threshold for considering a swab real-time RT-PCR-positive, by solely analysing swabs with an estimated ${ }^{10} \log$ virus titre greater than 1.6 or alternatively 2.6 (values are based on the titres of the standard curve).

\section{Results}

\subsection{Disease symptoms}

The unvaccinated turkeys (group 0 ) all showed symptoms of illness. Among the clinical signs were weight loss, paleness, fluffed-up feathers, slowness, an inability to stand, symptoms of palsy or the inability to keep the head straight on the body. Seven inoculated birds died or were euthanized within 5-8 days after challenge, and one inoculated bird was euthanized 18 days p.c. after showing severe nervous disorders. Nine contact turkeys died or were euthanized within 8-10 days p.c. Two inoculated and one contact turkey survived and stopped showing symptoms after 20/21 days p.c., after only showing fluffed-up feathers, weight loss, slowness and paleness.

One inoculated turkey from group 1 (vaccinated once) started showing lameness, inability to stand and palsy on 5 days p.c. and continued to show palsy until termination of the experiment. None of the other turkeys from group 1 or from group 2 (vaccinated twice) showed any disease related clinical symptoms.

\subsection{Serology}

Fig. 1 displays the $\mathrm{H} 7$ antibody titres which were determined with the HI test. The $\mathrm{HI}$ titres in group 1 were significantly $(p<0.0001)$ lower at the day of challenge than the HI titres in group 2 . Group 2 also seems to have a slightly faster response to the first vaccination when compared to group 1 ; the HI titre at 14 days post first vaccination was higher for group 2 than for group $1(p<0.01)$.

At day 28 p.c. the two inoculated and one contact infected surviving unvaccinated birds were positive in the IF test, indicating that these birds were fully challenged, and one inoculated bird of group 1 had a response at the threshold level of a positive test outcome. All other samples were negative.

\subsection{Virology}

Virus titres $\left({ }^{10} \mathrm{log}\right.$, expressed as equivalent units of $\operatorname{EID}_{50} / \mathrm{ml}$ ) in the tracheal and cloacal swabs of the unvaccinated turkeys were estimated from the quantitative real-time RT-PCR results, and shown in Fig. 2. The virus titres of the cloacal swabs were lower than those of the tracheal swabs within both the contact and inoculated groups, and viral RNA was also generally isolated during a shorter period from the cloacal swabs than from the tracheal swabs. Table 2 gives the qualitative results for the swabs of the unvaccinated birds. In Table 3 the results are given in numbers of
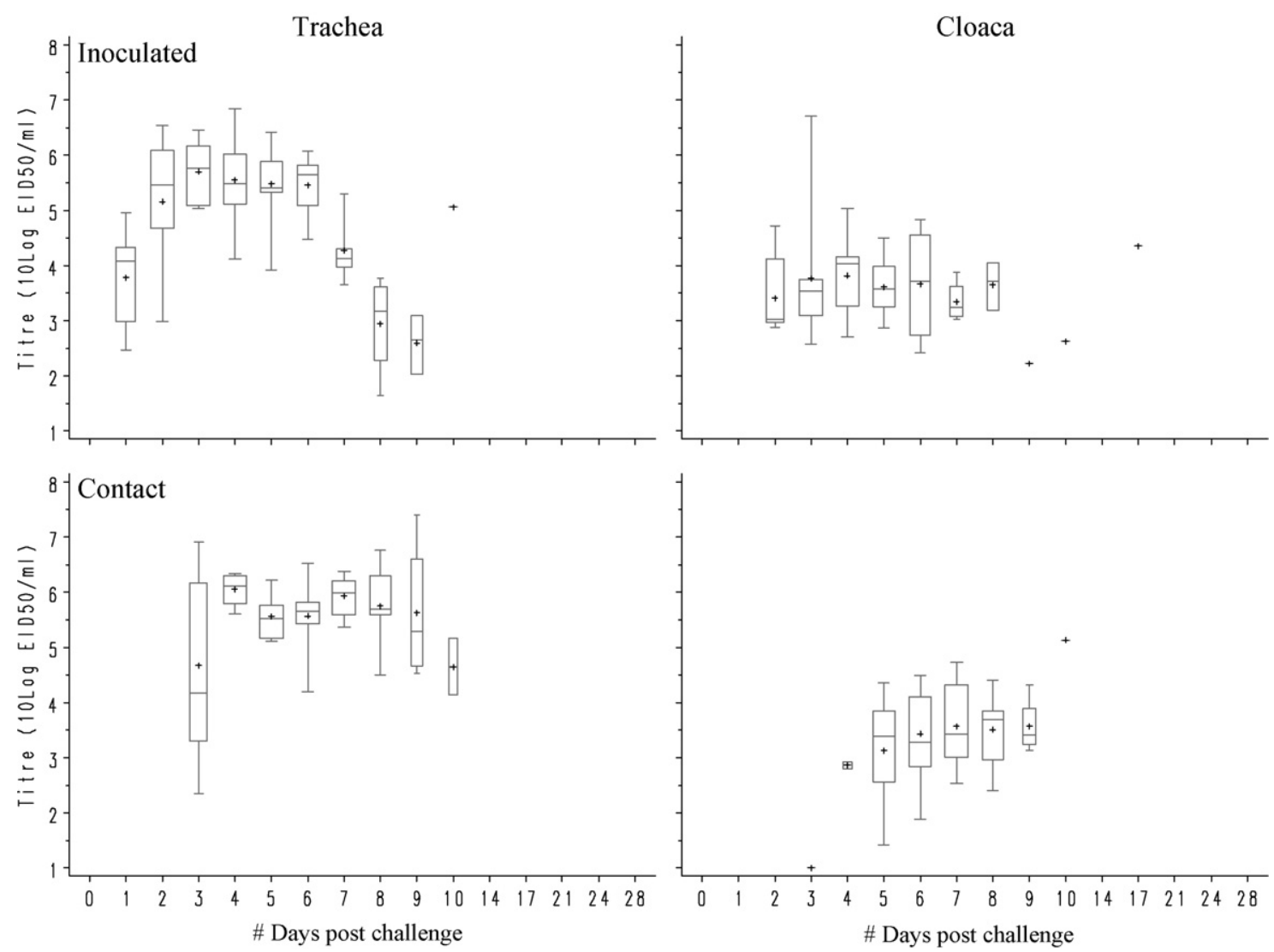

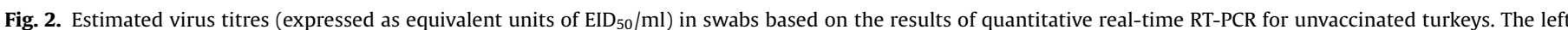

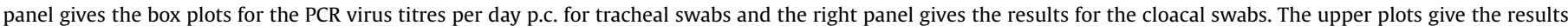

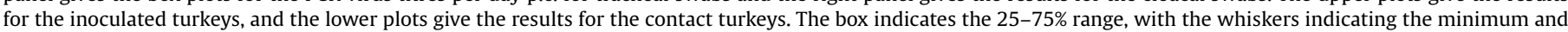

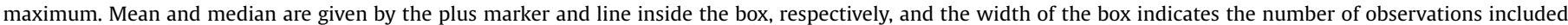
(maximum $=10$ ) 
Table 2

Qualitative results from real-time RT-PCR on tracheal and cloacal swabs for unvaccinated turkeys (group A/B) after challenge with HPAI H7N7 (at 12 weeks of age)

\begin{tabular}{|c|c|c|c|c|c|c|c|c|c|c|c|c|c|c|c|c|}
\hline \multirow[b]{2}{*}{ Group } & \multirow[b]{2}{*}{ Turkey } & \multicolumn{15}{|c|}{ Qualitative real-time RT-PCR results for trachea/cloaca swab at day post challenge } \\
\hline & & 1 & 2 & 3 & 4 & 5 & 6 & 7 & 8 & 9 & 10 & 14 & 17 & 21 & 24 & 28 \\
\hline \multirow[t]{10}{*}{ A } & I & $+/-$ & $+/-$ & $+/+$ & $+/+$ & $+/+$ & $+/+$ & $+/+$ & $+1+$ & $+/+$ & $-/+$ & $-/-$ & $-/-$ & $\dagger^{*}$ & & \\
\hline & I & $+/-$ & $+1+$ & $+1+$ & $+/+$ & $\dagger$ & & & & & & & & & & \\
\hline & I & $-/-$ & $+/-$ & $+/+$ & $+1+$ & $+1+$ & $+1+$ & $+1-$ & $+/-$ & $+1-$ & $+1-$ & $-/-$ & $-/-$ & $-/-$ & $-/-$ & $-/-$ \\
\hline & I & $-/-$ & $+/-$ & $+/+$ & $+/+$ & $+/+$ & $+/+$ & $+1+$ & $+/+$ & $+/-$ & $-/-$ & $-/-$ & $-1+$ & $-/-$ & $-/-$ & $-/-$ \\
\hline & I & $+/-$ & $+1+$ & $+/+$ & $+/+$ & $+/+$ & $+/+$ & $\dagger$ & & & & & & & & \\
\hline & $\mathrm{C}$ & nd & $-/-$ & $-/-$ & $+/-$ & $+/+$ & $+1+$ & $+/+$ & $+1+$ & $\dagger$ & & & & & & \\
\hline & $\mathrm{C}$ & nd & $-/-$ & $+/+$ & $+/-$ & $+/+$ & $+/+$ & $+/+$ & $\dagger$ & & & & & & & \\
\hline & $\mathrm{C}$ & nd & $-/-$ & $+/-$ & $+/+$ & $+1+$ & $+1+$ & $+1+$ & $+/+$ & $\dagger$ & & & & & & \\
\hline & $\mathrm{C}$ & nd & $-/-$ & $+/-$ & $+/-$ & $+/-$ & $+1+$ & $+/+$ & $+/+$ & $+1+$ & $\uparrow$ & & & & & \\
\hline & $\mathrm{C}$ & nd & $-1-$ & $+/-$ & $+/-$ & $+/+$ & $+/-$ & $+/ t$ & $+/+$ & $\dagger$ & & & & & & \\
\hline
\end{tabular}

B

\begin{tabular}{|c|c|c|c|c|c|c|c|c|c|c|c|c|c|c|}
\hline$+/-$ & $+/+$ & $+/+$ & $+/+$ & $+/+$ & $+/+$ & $\dagger$ & & & & & & & & \\
\hline$+/-$ & $+/+$ & $+/+$ & $+/+$ & $+/+$ & $+/+$ & $+/+$ & $\grave{\dagger}^{*}$ & & & & & & & \\
\hline$+/-$ & $+/+$ & $+/+$ & $+/+$ & $+/+$ & $+/+$ & $\dagger$ & & & & & & & & \\
\hline$+/-$ & $+/+$ & $+/+$ & $+/+$ & $+/+$ & $\dagger^{*}$ & & & & & & & & & \\
\hline$+1-$ & $+/+$ & $+/+$ & $+/+$ & $+/+$ & $+/+$ & $+/+$ & $+/+$ & $\dagger^{*}$ & & & & & & \\
\hline nd & $-/-$ & $+/-$ & $+/-$ & $+/+$ & $+/+$ & $+/+$ & $+/+$ & $+/+$ & $+/-$ & $-/-$ & $-/-$ & $-/-$ & $-/-$ & $-/-$ \\
\hline nd & $-/-$ & $+/-$ & $+/-$ & $+1+$ & $+/+$ & $+/+$ & $+/+$ & $\dagger$ & & & & & & \\
\hline nd & $-/-$ & $-/-$ & $+/-$ & $+/+$ & $+1+$ & $+/+$ & $+/+$ & $+/+$ & $\dagger$ & & & & & \\
\hline nd & $-/-$ & $-/-$ & $+/-$ & $+/+$ & $+/+$ & $+/+$ & $+/+$ & $+/+$ & $+/+$ & $\dagger^{*}$ & & & & \\
\hline nd & $-/-$ & $+/-$ & $+/+$ & $+/+$ & $+/+$ & $+/+$ & $+/+$ & $\dagger$ & & & & & & \\
\hline
\end{tabular}

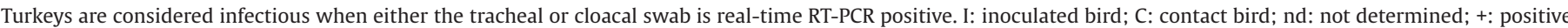
swab; -: negative swab; tracheal swab/cloacal swab; $\uparrow:$ turkey died; $\uparrow^{*}$ : turkey euthanized; dark grey: bird is case; light gray: bird is infectious.

Table 3

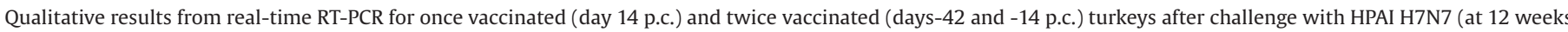
of age)

\begin{tabular}{|c|c|c|c|c|c|c|c|c|c|c|c|c|c|}
\hline \multirow[t]{2}{*}{ Group } & & \multicolumn{12}{|c|}{ \# real-time RT-PCR positive turkeys/\# turkeys per day post challenge } \\
\hline & & 1 & 2 & 3 & 4 & 5 & 6 & 7 & 8 & 9 & 10 & $\ldots$ & 28 \\
\hline \multirow[t]{2}{*}{ Vaccinated once (A) } & I & $0 / 5$ & $0 / 5$ & $0 / 5$ & $0 / 5$ & $0 / 5$ & $0 / 5$ & $0 / 5$ & $0 / 5$ & $0 / 5$ & $0 / 5$ & $\ldots$ & $0 / 5$ \\
\hline & $\mathrm{C}$ & nd & $0 / 5$ & $0 / 5$ & $0 / 5$ & $0 / 5$ & $0 / 5$ & $0 / 5$ & $0 / 5$ & $0 / 5$ & $0 / 5$ & $\ldots$ & $0 / 5$ \\
\hline \multirow[t]{2}{*}{ Vaccinated once (B) } & I & $1 / 5^{1}$ & $0 / 5$ & $0 / 5$ & $0 / 5$ & $0 / 5$ & $0 / 5$ & $0 / 5$ & $0 / 5$ & $0 / 5$ & $0 / 5$ & $\ldots$ & $0 / 5$ \\
\hline & $\mathrm{C}$ & nd & $0 / 5$ & $0 / 5$ & $0 / 5$ & $0 / 5$ & $0 / 5$ & $0 / 5$ & $0 / 5$ & $0 / 5$ & $0 / 5$ & $\ldots$ & $0 / 5$ \\
\hline \multirow[t]{2}{*}{ Vaccinated twice (A) } & I & $0 / 5$ & $0 / 5$ & $0 / 5$ & $0 / 5$ & $0 / 5$ & $0 / 5$ & $0 / 5$ & $0 / 5$ & $0 / 5$ & $0 / 5$ & $\ldots$ & $0 / 5$ \\
\hline & $\mathrm{C}$ & nd & $0 / 5$ & $0 / 5$ & $0 / 5$ & $0 / 5$ & $0 / 5$ & $0 / 5$ & $0 / 5$ & $0 / 5$ & $0 / 5$ & $\ldots$ & $0 / 5$ \\
\hline \multirow[t]{2}{*}{ Vaccinated twice (B) } & I & $0 / 5$ & $0 / 5$ & $0 / 5$ & $0 / 5$ & $0 / 5$ & $0 / 5$ & $0 / 5$ & $0 / 5$ & $0 / 5$ & $0 / 5$ & $\ldots$ & $0 / 5$ \\
\hline & C & nd & $0 / 5$ & $0 / 5$ & $0 / 5$ & $0 / 5$ & $0 / 5$ & $0 / 5$ & $0 / 5$ & $0 / 5$ & $0 / 5$ & $\ldots$ & $0 / 4^{2}$ \\
\hline
\end{tabular}

Turkeys are considered positive when either the tracheal or cloacal swab is real-time RT-PCR positive.

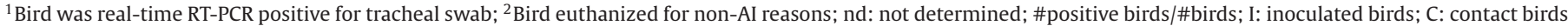

real-time RT-PCR positive birds (either a positive tracheal or cloacal swab) for treatment groups 1 and 2 . It can be seen that only in one inoculated bird from group 1 (vaccinated once) viral RNA was isolated from the tracheal swab at day 1 p.c. (this was not the bird that tested weakly positive in the IF test). One other inoculated turkey was positive for the tracheal swab at day 1 p.c., but this result could not be repeated and the swab was therefore considered negative. For treatment group 2 no positive swabs were found. In two contact turkeys tracheal swabs at day 8 and day 10 p.c. were weakly positive, however, again these positive results could not be repeated and therefore these swabs were considered negative.
The unvaccinated inoculated group had significantly more birds that shed virus than both vaccinated inoculated groups together $(p<0.0001)$.

\subsection{Transmission parameters}

It was only possible to perform statistical analysis on the unvaccinated groups, because in the vaccinated groups no positive swabs were found over multiple days. The mean infectious period in contact infected unvaccinated turkeys was 6.2 days (minimum 5 ; maximum 8 days). $\beta$ was estimated at 1.26 per infectious bird per day (95\% CI: $0.99-1.59$ ), which results in a $R_{0}$ of 7.8 (see Table 4 ). If

Table 4

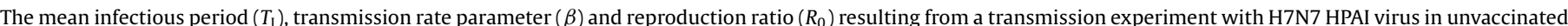
turkeys (challenged at 12 weeks of age), with different threshold values for a swab to be considered positive in the real-time RT-PCR

\begin{tabular}{|c|c|c|c|}
\hline Real-time RT-PCR positive when > & 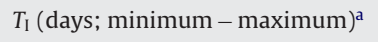 & $\beta(95 \%$ CI $)$ bird $^{-1}$ day $^{-1}$ & $R_{0}$ \\
\hline Lowest standard curve value per plate & $6.2(5-8)$ & $1.26(0.99-1.59)$ & 7.8 \\
\hline 1.6 & $6.3(5-9)$ & $1.29(1.05-1.60)$ & 8.1 \\
\hline 2.6 & $6.1(5-8)$ & 1.31 & 8.0 \\
\hline
\end{tabular}

a Contact infected turkeys. 
the threshold for considering a swab positive in real-time RT-PCR is set at a ${ }^{10} \log$ virus titre larger than 1.6 , the $\beta$ is 1.29 (1.05-1.60); if set at larger than 2.6 , it is 1.31 (because of equality of observations only a point estimate can be given).

\section{Discussion}

In the face of regularly emerging outbreaks of a variety of avian influenza viruses, vaccination might be a good tool in a control program, either prophylactic or as emergency measure. This study was conducted to measure the effect of vaccination with an H7N1 strain on the transmission of HPAI H7N7 virus in turkeys. Turkeys vaccinated once showed almost no virus shedding after challenge with H7N7 virus, and no transmission of the virus to vaccinated contact turkeys was observed. When the birds were vaccinated twice, no virus shedding was demonstrated at all after challenge, and hence transmission could not be quantified. Unvaccinated turkeys however, showed symptoms of illness, shed virus and were fully able to transmit the virus to susceptible contact turkeys. These results indicate that vaccination of turkeys could be useful as additional control measure to control an epidemic of HPAI as it was able to induce clinical protection and reduce virus shedding and virus transmission.

In the current study, real-time RT-PCR was used to detect viral RNA in swabs. Although the gold standard is virus isolation in eggs, real-time RT-PCR is supposed to be more sensitive as it may be able to pick up inactivated virus or virus neutralized by antibodies [19-21]. Consequently, it does not seem likely that the absence of virus shedding in vaccinated turkeys was caused by a lack of sensitivity. The virus might not be able to replicate because clearance of the virus is too fast due to the vaccination. A complete lack of virus shedding has also been observed in chickens vaccinated with the same vaccine and challenged with the same virus [7].

Hence, vaccination with H7N1 in turkeys was highly efficacious, a finding that seems in contrast to field observations and studies on the susceptibility of turkeys for an infection with AI strains. One possible explanation is the challenge model used. The route of infection and the challenge dose, however, were appropriate as challenge resulted in infection of all unvaccinated turkeys which developed AI symptoms, shed virus and transmitted the virus to pen mates. Therefore, it seems reasonable to assume that the challenge model was valid. Moreover, the dose used was in line with other experiments with turkeys, as carried out for example by Capua et al. [20]. Although they used a LPAI strain, it does not seem likely that a higher dose should have been used for HPAI H7N7. Remarkably, vaccination seemed to have been less effective when LPAI challenge was applied, as vaccinated turkeys still shed detectable, though reduced, amounts of virus after challenge $[20,22,23]$. Recent field experiences with LPAI also indicated that vaccinated turkeys could be infected [5]. In the field, infectious doses are expected to vary widely, but are generally assumed to be lower. It does not seem likely that the challenge dose in our experiment was too low.

Possibly, the age at vaccination might be of influence on the effect of vaccination. In Italy, meat turkeys were vaccinated at a younger age [5], but older birds are very well capable of mounting an immune response. The effect of age at vaccination on the efficacy of a vaccine needs some future research to provide practical guidelines. Other factors that generally may affect vaccine effectiveness in the field are climate, concurrent diseases, suboptimal dose at bird-level and suboptimal flock coverage.

Transmission studies can also be used for the development of monitoring programs based on $\beta$. It has been shown that turkeys are more susceptible to certain strains of LPAI viruses than chickens $[5,22]$ and also to H5N9 HPAI virus [13]. Furthermore, the inci- dence rate for HPAI H7N1 was greater for turkey farms than other bird species such as laying hens during the Italian epidemic in 1999-2000 [10]. A higher susceptibility however, does not necessarily mean a higher transmission. The same challenge virus, vaccine and protocol that were used in the current study, were also used in transmission experiments with (un)vaccinated chickens, teals and pheasants by Van der Goot et al. [7,8]. If we apply our statistical model on their results we find a $\beta$ of 1.72 per infectious chicken per day (95\% CI: 1.23-2.42), 2.71 (1.34-5.47) in pheasants and an unestimatable $\beta$ in teals. The estimated transmission rate parameter for turkeys based on our results was 1.26 (0.99-1.59), which did not differ significantly from the parameters found in other species. Therefore these findings do not support the assumption that turkeys have a higher HPAI H7N7 transmission. On the contrary, the findings suggest that HPAI H7N7 transmission in turkeys is lower. Pheasants still showed virus shedding and transmission after a single vaccination and in teals the virus was isolated from the inoculated vaccinated birds, which indicates that results in one bird species cannot be extrapolated to other bird species.

In conclusion it can be said that vaccination with $\mathrm{H} 7 \mathrm{~N} 1$ protects 12-week-old turkeys against challenge with H7N7 HPAI virus, and no transmission of the virus can therefore be measured in vaccinated turkeys. This study underlines the important role vaccination could have in controlling and preventing HPAI outbreaks.

\section{Acknowledgements}

The authors would like to thank the staff at CVI for their technical assistance, especially Sandra van de Water, John Voermans, Sylvia Pritz and Anita Jorna. Furthermore they would like to thank the animal care takers at CVI. Annemarie Bouma and two anonymous reviewers are gratefully acknowledged for providing valuable recommendations for the manuscript. This work was supported by EU Grants SSPE-CT-2004-513737 (HEALTHY POULTRY) and SSPECT-2005-022417 (FLUAID).

\section{References}

[1] Alexander DJ. An overview of the epidemiology of avian influenza. Vaccine 2007;25(30):5637-44.

[2] Ellis TM, Leung CYHC, Chow MKW, Bissett LA, Wong W, Guan Y, et al. Vaccination of chickens against H5N1 avian influenza in the face of an outbreak interrupts virus transmission. Avian Pathol 2004;33(4):405-12.

[3] Pfeiffer DU, Minh PQ, Martin V, Epprecht M, Otte MJ. An analysis of the spatial and temporal patterns of highly pathogenic avian influenza occurrence in Vietnam using national surveillance data. Vet J 2007;174:302-9.

[4] Villarreal-Chávez C, Rivera-Cruz E. An update on avian influenza in Mexico. Avian Dis 2003;47:1002-5.

[5] Capua I, Marangon S. The use of vaccination to combat multiple introductions of Notifiable Avian Influenza viruses of the H5 and H7 subtypes between 2000 and 2006 in Italy. Vaccine 2007;25(27):4987-95.

[6] Velthuis AGJ, Bouma A, Katsma WEA, Nodelijk G, De Jong MCM. Design and analysis of small-scale transmission experiments with animals. Epidemiol Infect 2007;135:202-17.

[7] Van der Goot JA, Koch G, De Jong MCM, Van Boven M. Quantification of the effect of vaccination on transmission of avian influenza (H7N7) in chickens. Proc Natl Acad Sci USA 2005;102(50):18141-6.

[8] Van der Goot JA, Van Boven M, Koch G, De Jong MCM. Variable effect of vaccination against highly pathogenic avian influenza (H7N7) virus on disease and transmission in pheasants and teals. Vaccine 2007;25(49):8318-25.

[9] Windhorst H-W. Changing regional patterns of turkey production and turkey meat trade. World's Poultry Sci J 2006;62(1):97-113.

[10] Mannelli A, Ferre N, Marangon S. Analysis of the 1999-2000 highly pathogenic avian influenza (H7N1) epidemic in the main poultry-production area in northern Italy. Prev Vet Med 2006;73(4):273-85.

[11] Max V, Herrera J, Moreira R, Rojas H. Avian influenza in Chile: a successful experience. Avian Dis 2006;51:363-5.

[12] Alexander DJ. A review of avian influenza in different bird species. Vet Microbiol 2000;74(1-2):3-13.

[13] Narayan O, Lang G, Rouse BT. A new influenza A virus infection in turkeys. IV. Experimental susceptibility of domestic birds to virus strain Turkey/Ontario 7732/1966. Arch Virol 1969;26(1):149-65. 
[14] Busani L, Dalla Pozza M, Bonfanti L, Toson M, Marangon S. Evaluation of the efficacy of intervention measures and vaccination for the control of LPAI epidemics in Verona province (Veneto, Italy). Avian Dis 2007;51:463-6.

[15] Council Directive 92/40/EEC. Off J Eur Communities 1992;L167:1-16.

[16] Cattoli G, Terregino C, Brasola V, Rodriguez JF, Capua I. Development and preliminary validation of an ad hoc N1-N3 discriminatory test for the control of avian influenza in Italy. Avian Dis 2003;47:1060-2.

[17] Stegeman JA, Bouma A, Elbers ARW, De Jong MCM, Nodelijk G, De Klerk F et al. Avian influenza A virus (H7N7) epidemic in The Netherlands in 2003: course of the epidemic and effectiveness of control measures. J Infect Dis 2004;190(12):2088-95

[18] Van der Goot JA, De Jong MCM, Koch G, Van Boven M. Comparison of the transmission characteristics of low and high pathogenicity avian influenza A virus (H5N2). Epidemiol Infect 2003;131(2):1003-13.

[19] Spackman E, Senne DA, Myers TJ, Bulaga LL, Garber LP, Perdue ML, et al. Development of a Real-Time reverse transcriptase PCR assay for type A influenza virus and the avian $\mathrm{H} 5$ and $\mathrm{H} 7$ hemagglutinin subtypes. J Clin Microbiol 2002;40(9):3526-4260.

[20] Capua I, Terregino C, Cattoli G, Toffan A. Increased resistance of vaccinated turkeys to experimental infection with an H7N3 low-pathogenicity avian influenza virus. Avian Pathol 2004;33(2):158-63.

[21] Lee C-W, Suarez DL. Application of real-time RT-PCR for the quantitation and competitive replication study of $\mathrm{H} 5$ and $\mathrm{H} 7$ subtype avian influenza virus. Virol Methods 2004;119(2):151-8.

[22] Tumpey TM, Kapczynski DR, Swayne DE. Comparative susceptibility of chickens and turkeys to avian influenza A H7N2 virus infection and protective efficacy of a commercial avian influenza H7N2 virus vaccine. Avian Dis 2004: $167-76$.

[23] Kodihalli S, Sivanandan V, Nagaraja KV, Shaw D, Halvorson DA. A type-specific avian influenza virus subunit vaccine for turkeys: induction of protective immunity to challenge infection. Vaccine 1994;12(15):1467-72. 